\title{
Evaluation of Drugs with Therapeutic Potential for Susceptibility of Neisseria Gonorrhoeae Isolates from 8 Provinces in China from 2018
}

This article was published in the following Dove Press journal: Infection and Drug Resistance

\author{
Xiao-Li Zheng ${ }^{1,2}$ \\ Wen-Qi Xu ${ }^{1,2}$ \\ Jing-Wei Liu (iD) ${ }^{1,2}$ \\ Xiao-Yu Zhu ${ }^{1,2}$ \\ Shao-Chun Chen (D) ${ }^{1,2}$ \\ Yan Han ${ }^{1,2}$ \\ Xiu-Qin Dai ${ }^{1,2}$ \\ Isabelle Griffin Goodman (iD ${ }^{3}$ \\ Christoph Budjan (iD) 4,5 \\ Xiang-Sheng Chen (iD) ${ }^{1,2}$ \\ Yue-Ping Yin (iD) 1,2 \\ 'Institute of Dermatology and Hospital \\ for Skin Diseases, Chinese Academy of \\ Medical Sciences and Peking Union \\ Medical College, Nanjing, People's \\ Republic of China; ${ }^{2}$ National Center for \\ Sexually Transmitted Diseases Control, \\ Chinese Center for Disease Control and \\ Prevention, Nanjing, People's Republic of \\ China; ${ }^{3}$ Boston University, Boston, MA, \\ USA; ${ }^{4}$ Department of Data Sciences, \\ Dana-Farber Cancer Institute, Boston, \\ MA, USA; ${ }^{5}$ Department of Systems \\ Biology, Harvard Medical School, Boston, \\ MA, USA
}

Correspondence: Yue-Ping Yin Institute of Dermatology and Hospital for Skin Diseases, Chinese Academy of Medical Sciences and Peking Union Medical College, No. 12, Jiangwangmiao Street, Nanjing 210042, People's Republic of China

Tel/Fax +862585478024

Email yinyp@ncstdlc.org
Purpose: The study aimed to evaluate meropenem, fosfomycin, berberine hydrochloride, and doxycycline minimum inhibitory concentrations (MICs) of Neisseria gonorrhoeae collected from eight provinces in China in 2018.

Methods: The MICs of 540 Neisseria gonorrhoeae isolates (451 isolates selected randomly and 89 isolates selected with preference) were determined to meropenem, fosfomycin, berberine hydrochloride, and doxycycline using the agar dilution method, and the MICs of ceftriaxone and azithromycin were detected for comparison.

Results: Among 451 randomly selected isolates, the $\mathrm{MIC}_{90}$ was $0.06 \mathrm{mg} / \mathrm{L}$ for meropenem, $64 \mathrm{mg} / \mathrm{L}$ for fosfomycin, $64 \mathrm{mg} / \mathrm{L}$ for berberine hydrochloride, and $16 \mathrm{mg} / \mathrm{L}$ for doxycycline. All isolates showed the MIC $\leq 0.125 \mathrm{mg} / \mathrm{L}$ to meropenem, 13 isolates $(2.9 \%)$ showed $\mathrm{MIC}>$ $64 \mathrm{mg} / \mathrm{L}$ to fosfomycin, 8 isolates $(1.8 \%)$ demonstrated MIC $>64 \mathrm{mg} / \mathrm{L}$ to berberine hydrochloride, and 271 isolates $(60.1 \%)$ demonstrated MIC $>1 \mathrm{mg} / \mathrm{L}$ to doxycycline. Comparing all 540 tested isolates, a correlation of $r=0.50(P<0.001)$ between meropenem and ceftriaxone MIC was observed. In 24 ceftriaxone-decreased susceptibility isolates, all isolates showed an $\mathrm{MIC} \leq 0.125 \mathrm{mg} / \mathrm{L}$ for meropenem, 1 isolate $(4.2 \%)$ showed an $\mathrm{MIC}>64 \mathrm{mg} / \mathrm{L}$ for fosfomycin, 1 isolate $(4.2 \%)$ showed an $\mathrm{MIC}>64 \mathrm{mg} / \mathrm{L}$ for berberine hydrochloride, and 13 isolates $(54.2 \%)$ showed an $\mathrm{MIC}>1 \mathrm{mg} / \mathrm{L}$ for doxycycline. In 87 azithromycin resistant isolates, all isolates showed an MIC $\leq 0.125 \mathrm{mg} / \mathrm{L}$ for meropenem, 2 isolates $(2.3 \%)$ showed an $\mathrm{MIC}>64 \mathrm{mg} / \mathrm{L}$ for fosfomycin, 4 isolates $(4.6 \%$ ) showed an MIC $>64 \mathrm{mg} / \mathrm{L}$ for berberine hydrochloride, and 64 isolates $(73.6 \%)$ showed an MIC $>1 \mathrm{mg} / \mathrm{L}$ for doxycycline.

Conclusion: The in vitro results suggest that meropenem might be a promising treatment option for resistant gonococcal infections, while the effects of fosfomycin and berberine hydrochloride should be further evaluated as potential therapeutic agents. The effectiveness of these drugs in animal experiments and clinical use may need further study.

Keywords: Neisseria gonorrhoeae, treatment, antimicrobial resistance, meropenem, fosfomycin

\section{Introduction}

Neisseria gonorrhoeae ( $N$. gonorrhoeae), a commonly reported sexually transmitted pathogen, presents a global public health threat with total incident cases approximating 86.9 million. ${ }^{1}$ In 2019 alone, 117,938 new gonorrhea cases were reported in China. ${ }^{2}$ Currently, ceftriaxone is the recommended gonorrhea treatment in China, ${ }^{3}$ while ceftriaxone and azithromycin are recommended as the first-line therapeutic treatment in America, ${ }^{4}$ Australia, ${ }^{5}$ Canada, ${ }^{6}$ Europe, ${ }^{7}$ New Zealand, ${ }^{8}$ 
and by the World Health Organization(WHO). ${ }^{9}$ However, $N$. gonorrhoeae has developed resistance to all previously used antimicrobial therapies including azithromycin and furthermore, it shows decreased susceptibility to ceftriaxone(DSC, defined as isolates with ceftriaxone $\mathrm{MIC} \geq 0.25 \mathrm{mg} / \mathrm{L}$ in China currently $)^{10-12}$ which poses a significant global healthcare burden.

The WHO carried out a global action plan to control the spread of antimicrobial-resistant $N$. gonorrhoeae and minimize its impact on the population. ${ }^{13}$ In response to rising gonococcal antimicrobial resistance, the National Health Commission's Committee of Experts on Rational Drug Use classified gonococcal antimicrobial resistance as part of the highest priority category for drug resistance surveillance in China, and the China National Action Plan to Contain Antimicrobial Resistance was jointly issued by 14 government sectors in $2016 .{ }^{14}$ In light of dwindling treatment options and increasing antibiotic resistance of $N$. gonorrhoeae, the ROADMAP plan was carried out to address research needs for $N$. gonorrhoeae antimicrobial resistance in China. ${ }^{15}$ Treatment of gonorrhea is a global health concern that requires immediate attention. Given the limited antibiotic arsenal, finding putative alternative therapeutic drugs has become an urgent priority in global public health.

There are constant efforts to develop new antimicrobial drugs, such as zoliflodacin and solithromycin, but such novel antimicrobial development is often cumbersome. A common alternative to this is to simply repurpose available drugs for the treatment of specific infections. With broad-spectrum antimicrobial activities, an in vitro study found meropenem to be a promising alternative therapeutic agent for cephalosporin-resistant $N$. gonorrhoeae with a mosaic penA allele, whereas other carbapenems and faropenem had a lower potential for effective treatment. ${ }^{16}$ Fosfomycin also has been suggested as a possible alternative antibiotic against $N$. gonorrhoeae both in vitro and in vivo. ${ }^{17,18}$ Previously, in vitro studies have demonstrated that fosfomycin can retain activity against ceftriaxone-resistant $N$. gonorrhoeae. $^{17}$ Moreover, a recent randomized controlled clinical trial of the use of fosfomycin trometamol to treat uncomplicated gonococcal urethritis in men exhibited excellent efficacy in China. ${ }^{18}$ Additionally, berberine, a plant alkaloid with a long history of medicinal use in both Ayurvedic and Chinese medicine has been reported to be active against grampositive and gram-negative bacteria with an unclear antibacterial mechanism. ${ }^{19}$ Berberine is a multi-target compound which inhibits arylamine $\mathrm{N}$-acetyltransferase activity in
Salmonella typhi, ${ }^{20}$ targets assembly of Escherichia coli cell division protein FtsZ, ${ }^{21,22}$ blocks adhesion of Escherichia coli, and suppresses the synthesis and assembly of fimbriae. ${ }^{23}$ A clinical study investigating treatment options for Chlamydia trachomatis infection treated one group with aqueous berberine chloride eye drops. An examination of the patients' conjunctival scrapings showed no lingering Chlamydia trachomatis infection after the treatment, and no relapses occurred within 1 year of follow-up examinations. $^{24,25}$ As for doxycycline, the Chinese Guidelines for diagnosis and treatment of syphilis, gonorrhea, and genital Chlamydia trachomatis infection (2020) recommend doxycycline to treat gonorrhea that does not exclude chlamydia infection or gonococcal pelvic inflammatory disease. The 2015 USA Sexually Transmitted Diseases Treatment Guidelines proposed that doxycycline could replace azithromycin as a second antimicrobial to be used in combination with ceftriaxone or cefixime in the case of azithromycin allergy. ${ }^{4}$ While the wide variety of uses of these drugs has been investigated at length, there are very limited data on the use of such drugs at mitigating $N$. gonorrhoeae susceptibility in China. In this study, we evaluated the MICs of the putative anti- $N$. gonorrhoeae drugs, ceftriaxone, and azithromycin with $N$. gonorrhoeae isolates newly collected from eight provinces of China in 2018 and we described the MIC distributions in different provinces. We then performed a correlation analysis between these drugs and ceftriaxone or azithromycin to evaluate the cross-resistance. Furthermore, we evaluated the performance of evaluated drugs in DSC or azithromycin resistant isolates.

\section{Materials and Methods Ethical Approval}

The ethics (2014-LS-026) of this research were approved by the Medical Ethics Committee at the Institute of Dermatology and Hospital for Skin Diseases, the Chinese Academy of Medical Sciences \& Peking Union Medical College, and the National Center for Sexually Transmitted Disease Control.

\section{Drugs}

Meropenem, fosfomycin, doxycycline, ceftriaxone and azithromycin were obtained from United States Pharmacopeia (USP) and berberine hydrochloride was obtained from Chengdu Must Biotechnology Co., Ltd (Chengdu, China). 


\section{Gonococcal Isolates}

Clinical isolates were collected in outpatient departments from eight provinces (Beijing, Guangdong, Guangxi, Liaoning, Sichuan, Shaanxi, Tianjin, and Yunnan) which were included in China Gonococcal Resistance Surveillance Programme (China-GRSP) from January 1 to December 31, 2018. Samples were inoculated in selective Thayer-Martin (TM) medium and cultured at $36-36.5^{\circ} \mathrm{C}$ supplemented with $5 \% \mathrm{CO}_{2}$ humidified atmosphere for 24 to $48 \mathrm{hrs}$. Then, the culture-positive presumptive $N$. gonorrhoeae isolates were identified, preserved, and transferred as previously described. ${ }^{10}$ In total, 540 isolates from Beijing, Guangdong, Guangxi, Liaoning, Sichuan, Shaanxi, Tianjin and Yunnan were selected for the determination of antimicrobial susceptibility. In this study, 451 isolates were selected without preference to evaluate the sensitivity of these drugs in different provinces, and 89 isolates were selected with preference (isolates with relatively high MICs of ceftriaxone or azithromycin) to further increase the number of resistant isolates for cross-resistance analysis.

\section{Antimicrobial Susceptibility Test}

Antimicrobial susceptibility tests to meropenem, fosfomycin, berberine hydrochloride, doxycycline, ceftriaxone, and azithromycin were determined for all isolates using the agar dilution method. ${ }^{10}$ Firstly, isolates were inoculated from frozen stocks onto selective TM medium, and sub-cultured on GC agar base medium (Oxide, Hampshire, England) with 10\% defibrinated sheep blood (Bianzhen Biotechnology, Nanjing, China) at $36^{\circ} \mathrm{C}$ $5 \% \mathrm{CO}_{2}$-enriched and humidified atmosphere for 18 to $20 \mathrm{hrs}$. With these culture positive isolates, morphology identification and oxidase tests were repetitively finished before antimicrobial susceptibility tests were performed. Then, suspensions of $10^{7}$ bacteria per milliliter were prepared by adding scraped colonies into sterile saline solution (Pengyao Pharmacy, Wuxi, China), and suspensions ( $10^{4}$ per inoculation) were added to the culture medium (GC agar base medium supplemented with $10 \%$ defibrinated sheep blood) containing antibiotics on a $10 \mathrm{~cm}$ square plate using a multipoint inoculator. Next, the plates were incubated at $36^{\circ} \mathrm{C}$ humidified $5 \% \mathrm{CO}_{2}$-enriched atmosphere for 18 to $24 \mathrm{hrs}$, and the $N$. gonorrhoeae growth in plates containing various antibiotic concentrations was observed and recorded. The concentrations were $0.004,0.008,0.015,0.03,0.06,0.125$, $0.25,0.5$, and $1 \mathrm{mg} / \mathrm{L}$ for meropenem, $1,2,4,8,16,32,64$, and $128 \mathrm{mg} / \mathrm{L}$ for fosfomycin, $1,2,4,8,16,32,64$, and $128 \mathrm{mg} / \mathrm{L}$ for berberine hydrochloride, $0.125,0.25,0.5,1,2,4,8,16,32$, and $64 \mathrm{mg} / \mathrm{L}$ for doxycycline, $0.008,0.015,0.03,0.06,0.125,0.25$, 0.5 , and $1 \mathrm{mg} / \mathrm{L}$ for ceftriaxone, and $0.03,0.06,0.125,0.25,0.5$, 1,2 , and $4 \mathrm{mg} / \mathrm{L}$ for azithromycin. For the fosfomycin susceptibility test, culture medium was additionally supplemented with 25 mg/L glucose-6-phosphate (Sigma, Saint Louis, USA). Here, the lowest concentration of the antibiotic that inhibited isolates growth was defined as MIC. Interpretive criteria were in accordance with Clinical and Laboratory Standards Institute (CLSI) M100-S30 to categorize profiles of susceptibility and resistance. There are no standardized criteria in meropenem, fosfomycin, berberine hydrochloride, and doxycycline for $N$. gonorrhoeae treatment through EUCAST and CLSI methodologies so far. According to CLSI interpretive criteria, MIC interpretation is shown in Table 1. For experimental quality assurance, five WHO $N$. gonorrhoeae reference isolates $(\mathrm{G}, \mathrm{J}, \mathrm{K}, \mathrm{L}$, and $\mathrm{P})$ and ATCC49226 were used as controls. ${ }^{26}$

\section{Data Analysis}

If the MIC of reference isolates in every batch of tests was same as standard or fluctuated within 1 MIC dilution, this batch of test was included in the data analysis. Four hundred and fifty-one isolates selected without preference from eight

Table I Antimicrobial Susceptibility Testing Results for 45I Clinical N. Gonorrhoeae Isolates and Breakpoints Used in This Study

\begin{tabular}{|c|c|c|c|c|c|c|c|c|}
\hline \multirow[t]{2}{*}{$\begin{array}{l}\text { Antimicrobial } \\
\text { Agent }\end{array}$} & \multicolumn{4}{|l|}{ MIC (mg/L) } & \multicolumn{3}{|c|}{$\begin{array}{l}\text { MIC Interpretation Used for Analysis in this } \\
\text { Study (mg/L) }\end{array}$} & \multirow[t]{2}{*}{$\begin{array}{l}\text { Percentage of } \\
\text { Resistant Isolates }\end{array}$} \\
\hline & Range & Mode & $\mathrm{MIC}_{50}$ & $M I C_{90}$ & Susceptible & $\begin{array}{l}\text { Intermediately } \\
\text { Susceptible }\end{array}$ & Resistant & \\
\hline Meropenem $*^{* a}$ & $\leq 0.004-0.125$ & 0.03 & 0.03 & 0.06 & $\leq 1$ & 2 & $\geq 4$ & 0 \\
\hline Fosfomycin ${ }^{\mathrm{b}}$ & $4-\geq 256$ & 32 & 32 & 64 & $\leq 64$ & 128 & $\geq 256$ & 0.2 \\
\hline $\begin{array}{l}\text { Berberine } \\
\text { hydrochloride }\end{array}$ & $2-\geq 256$ & 16 & 16 & 64 & NA & NA & NA & - \\
\hline Doxycycline $^{d}$ & $\leq 0.125-64$ & 2 & 2 & 16 & $\leq 0.25$ & $0.5-1$ & $\geq 2$ & 60.1 \\
\hline
\end{tabular}

Notes: *4I5 isolates had efficient MIC results for meropenem. ${ }^{a}$ The MIC interpretation of meropenem according to CLSI breakpoint in testing and reporting for Escherichia

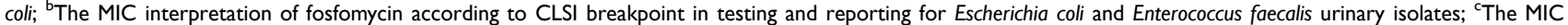
interpretation of Berberine hydrochloride is not available; ${ }^{\mathrm{d}}$ The MIC interpretation of doxycycline according to CLSI breakpoint in tetracycline for N. gonorrhoeae. Abbreviation: NA, not available. 
provinces were used to evaluate the MIC level in China. All other analyses were carried out among all 540 isolates. Here, we describe the distribution of collected $N$. gonorrhoeae isolates MICs to meropenem, fosfomycin, berberine hydrochloride and doxycycline, and calculated antimicrobial resistance percentages referring to CLSI breakpoint in other bacteria or breakpoint of similar drugs in $N$. gonorrhoeae. Comparison of individual provinces and nationwide susceptibility profiles were performed by Chi-square statistics or Fisher exact test when appropriate. A $P$-value $<0.05$ was considered statistically significant. Correlation between MICs of evaluated drugs and ceftriaxone or azithromycin was determined by linear regression of $\log _{2}$-transformed MIC data among 540 isolates. Through the correlation coefficient $r$, possible crossresistance between evaluated drugs and ceftriaxone or azithromycin was determined. For MICs determined as $\leq$ or $\geq$ a specific value, this specific value was used for calculating linear regression of $\log _{2}$-transformed MIC data. Moreover, the MICs distribution in DSC or azithromycin resistant isolates was analysed. Statistical analyses were carried out using Excel (Microsoft, Washington, USA), SPSS software (IBM, New York, USA), and GraphPad Prism software (GraphPad
Software, San Diego, CA, USA). Figures were made using $\mathrm{R}$ software (GNU System), PowerPoint (Microsoft, Washington, USA), and Adobe Photoshop CS5 software (Adobe Systems Incorporated, CA, USA).

\section{Results}

\section{Distribution and Anatomical Sites of N. gonorrhoeae Isolates}

Geographically, the 451 isolates were collected from 8 provinces (Beijing, Guangdong, Guangxi, Liaoning, Sichuan, Shaanxi, Tianjin and Yunnan) and the number of isolates in each province is shown in Figure 1, which showed the geographical diversity of the sample. The specific numbers of isolates for each province were 53, 59, 76, 38, 67, 52, 48 and 58 , respectively. The anatomical sites of the 451 isolates were from genitourinary tract.

\section{Antimicrobial Susceptibility of 45I Isolates}

The range, mode, $\mathrm{MIC}_{50}, \mathrm{MIC}_{90}$, $\mathrm{MIC}$ interpretation, and percentage of isolates resistant to meropenem, fosfomycin,

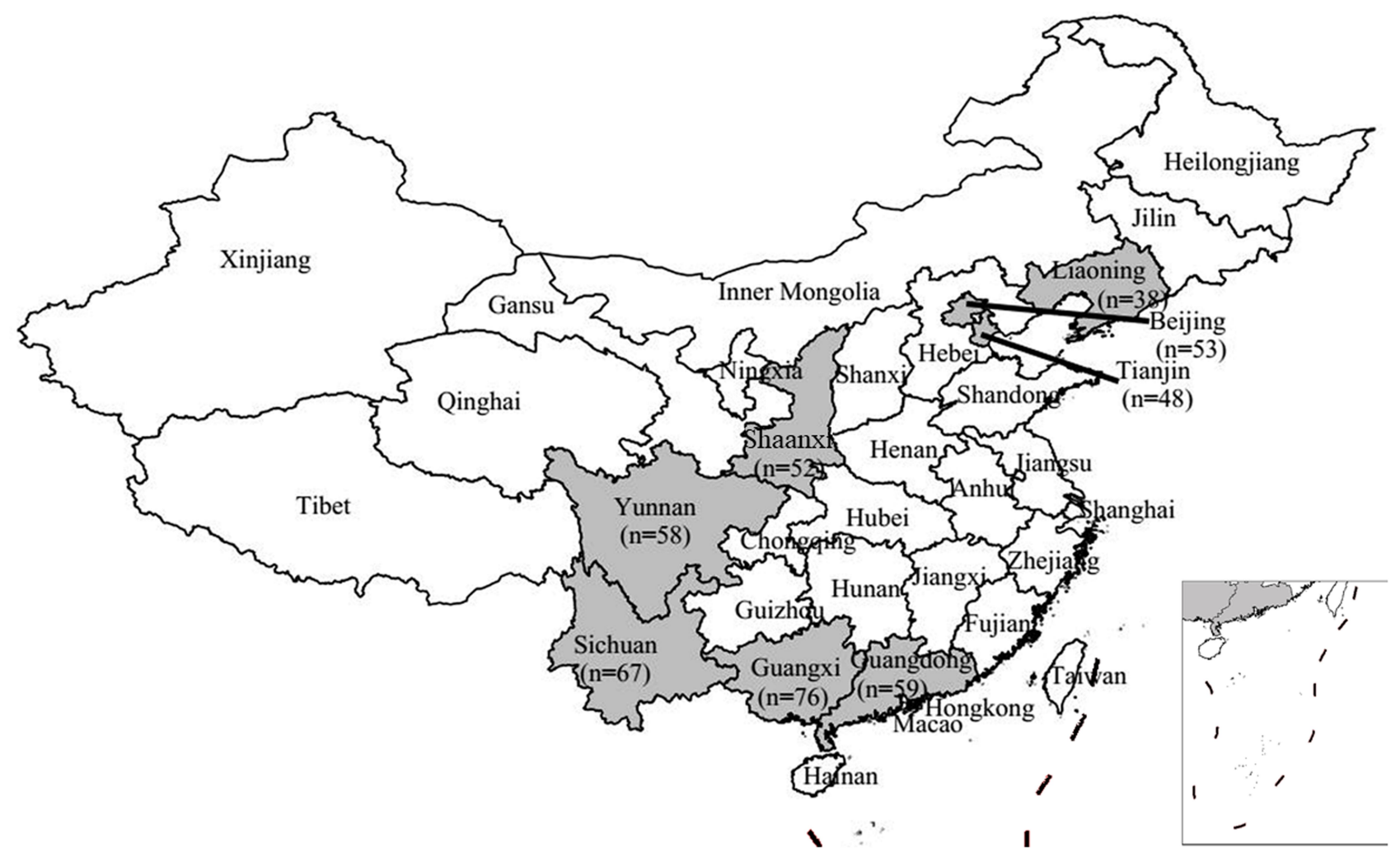

Figure I Geographic locations of the provinces where $45 \mathrm{I} \mathrm{N}$. gonorrhoeae isolates for antimicrobial susceptibility tests were collected and the number of isolates collected from each province.

Abbreviation: $n$, number. 

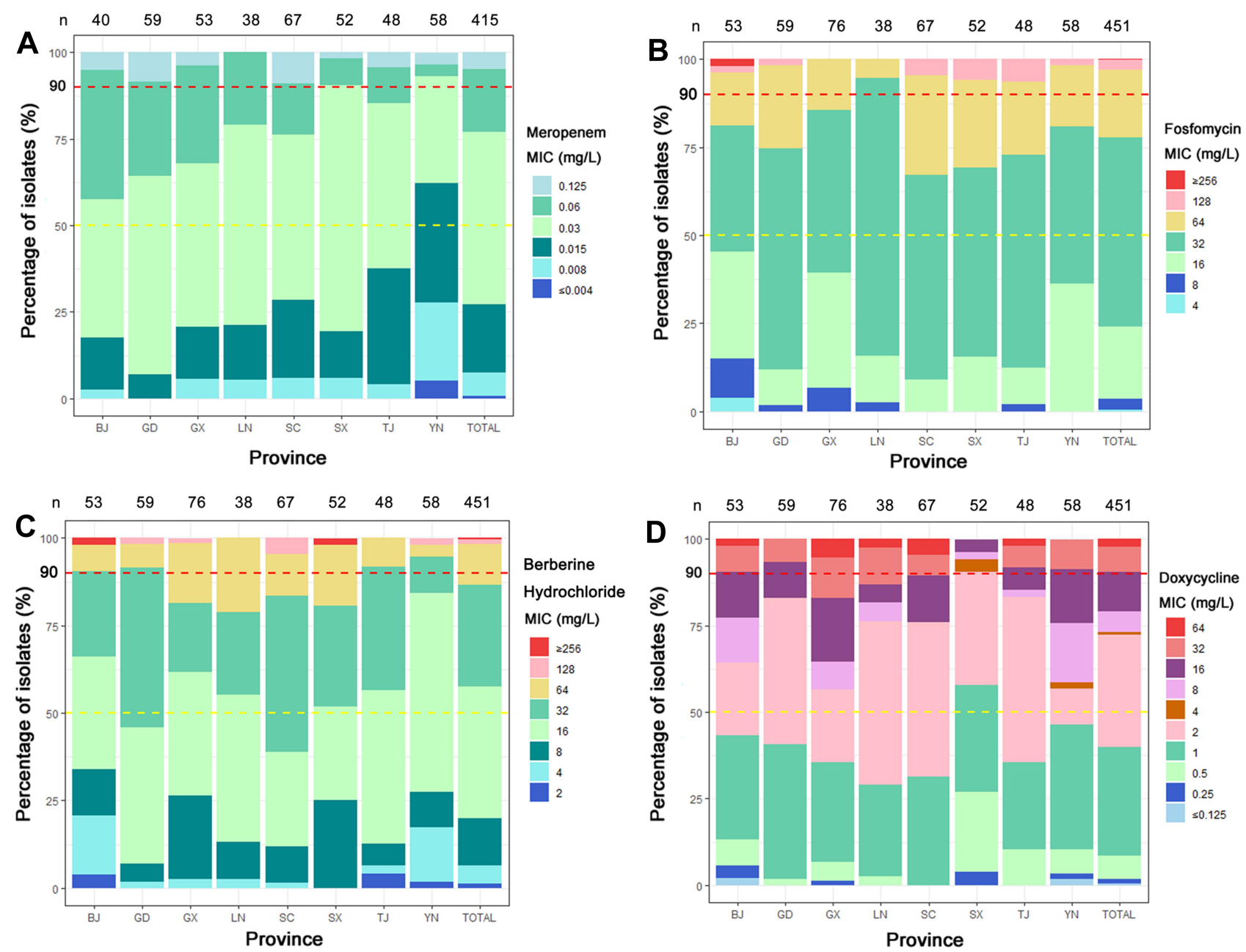

Figure 2 The percentage of $N$. gonorrhoeae isolates with different MICs (mg/L) for four evaluated agents, by province. (A) meropenem; (B) fosfomycin; (C) berberine hydrochloride; (D) doxycycline. The $\mathrm{MIC}_{50}$ (yellow-dotted line), $\mathrm{MIC}_{90}$ (red-dotted line) and number of isolates per province was shown in each Figure.

Abbreviations: BJ, Beijing; GD, Guangdong; GX, Guangxi; LN, Liaoning; SC, Sichuan; SX, Shaanxi; TJ, Tianjin; YN, Yunnan; n, number.

berberine hydrochloride, and doxycycline are shown in Table 1. In this study, 415 of 451 isolates $(92.0 \%)$ had MIC results for meropenem, while all isolates have MICs for fosfomycin, berberine hydrochloride, doxycycline, ceftriaxone and azithromycin.

As shown in Table 1, the MIC range for meropenem was $\leq 0.004-0.125 \mathrm{mg} / \mathrm{L}$. The mode, $\mathrm{MIC}_{50}$, and $\mathrm{MIC}_{90}$ were $0.03,0.03$, and $0.06 \mathrm{mg} / \mathrm{L}$, respectively. Twenty of 415 isolates (4.8\%) exhibited MIC of $0.125 \mathrm{mg} / \mathrm{L}$. Of the 415 isolates, all isolates demonstrated $\mathrm{MIC} \leq 0.125 \mathrm{mg} / \mathrm{L}$ to meropenem. The percentage of the isolates with different MICs of meropenem by provinces are shown in Figure $2 \mathrm{~A}$. Both the mode and $\mathrm{MIC}_{50}$ were the same among tested provinces with an overall level of $0.03 \mathrm{mg} / \mathrm{L}$, except for Yunnan with $0.015 \mathrm{mg} / \mathrm{L}$ (Figure 2A). Similarly, $\mathrm{MIC}_{90}$ were alike with an overall level of
$0.06 \mathrm{mg} / \mathrm{L}$, except Shaanxi and Yunnan with $0.03 \mathrm{mg} / \mathrm{L}$ (Figure 2A).

Fosfomycin MICs ranged from 4 to $\geq 256 \mathrm{mg} / \mathrm{L}$, and the mode, $\mathrm{MIC}_{50}$, and $\mathrm{MIC}_{90}$ were $32 \mathrm{mg} / \mathrm{L}, 32 \mathrm{mg} / \mathrm{L}$, and $64 \mathrm{mg} / \mathrm{L}$ (Table 1). The percentage of isolates $\mathrm{MIC} \leq$ $64 \mathrm{mg} / \mathrm{L}$ to fosfomycin were $97.1 \%$ (438/451), and 12 isolates $(2.7 \%)$ showed $\mathrm{MIC}=128 \mathrm{mg} / \mathrm{L}$, meanwhile 1 isolate $(0.2 \%)$ showed $\mathrm{MIC} \geq 256 \mathrm{mg} / \mathrm{L}$. Isolates with different MICs of fosfomycin are shown by provinces in Figure $2 \mathrm{~B}$. Both the mode and $\mathrm{MIC}_{50}$ in provinces were $32 \mathrm{mg} / \mathrm{L}$ (Figure 2B). The overall $\mathrm{MIC}_{90}$ was $64 \mathrm{mg} / \mathrm{L}$, which was also found in all provinces, except Liaoning with $32 \mathrm{mg} / \mathrm{L}$ (Figure 2B). The proportion of $\mathrm{MIC} \geq$ $128 \mathrm{mg} / \mathrm{L}$ varied from $0 \%$ to $5.8 \%$ in different provinces.

Berberine hydrochloride $\mathrm{MICs}$ ranged from 2 to $\geq 256 \mathrm{mg} / \mathrm{L}$, and the mode, $\mathrm{MIC}_{50}$, and $\mathrm{MIC}_{90}$ were 
$16 \mathrm{mg} / \mathrm{L}, 16 \mathrm{mg} / \mathrm{L}$, and $64 \mathrm{mg} / \mathrm{L}$, respectively (Table 1 ). Eight of 451 isolates (1.8\%) demonstrated MIC $\geq 128 \mathrm{mg} /$ L. Isolates with different MICs of berberine hydrochloride are shown by province in Figure 2C. Both the mode and $\mathrm{MIC}_{50}$ in the provinces were $16 \mathrm{mg} / \mathrm{L}$, except mode in Guangdong, Sichuan and Shaanxi was $32 \mathrm{mg} / \mathrm{L}, \mathrm{MIC}_{50}$ in Guangdong and Sichuan was $32 \mathrm{mg} / \mathrm{L}$ (Figure 2C). The $\mathrm{MIC}_{90}$ in Guangxi, Liaoning, Sichuan, and Shaanxi was $64 \mathrm{mg} / \mathrm{L}$, while that in Beijing, Guangdong, Tianjin, and Yunnan was $32 \mathrm{mg} / \mathrm{L}$ (Figure 2C). The proportion of MIC $\geq 128 \mathrm{mg} / \mathrm{L}$ varied from $0 \%$ to $4.5 \%$ in different provinces.

Doxycycline MICs ranged from $\leq 0.125$ to $64 \mathrm{mg} / \mathrm{L}$, and the mode, $\mathrm{MIC}_{50}$ and $\mathrm{MIC}_{90}$ were $2 \mathrm{mg} / \mathrm{L}, 2 \mathrm{mg} / \mathrm{L}$ and $16 \mathrm{mg} / \mathrm{L}$ (Table 1), respectively. Of 451 isolates, 180 isolates $(39.9 \%)$ exhibited $\mathrm{MIC} \leq 1 \mathrm{mg} / \mathrm{L}$ to doxycycline, and 271 isolates $(60.1 \%)$ demonstrated MIC $\geq 2 \mathrm{mg} / \mathrm{L}$. The proportions of the isolates with different MICs of doxycycline by province are shown in Figure 2D. The mode in provinces was $2 \mathrm{mg} / \mathrm{L}$, except Beijing, Guangxi and Yunnan with $1 \mathrm{mg} / \mathrm{L}$. The $\mathrm{MIC}_{50}$ in provinces was $2 \mathrm{mg} /$ $\mathrm{L}$, except for Shaanxi (1 mg/L) (Figure 2D). The $\mathrm{MIC}_{90}$ in provinces was $16 \mathrm{mg} / \mathrm{L}$, except in Guangxi $(32 \mathrm{mg} / \mathrm{L})$, Liaoning (32 mg/L), Sichuan (32 mg/L) and Shaanxi $(2 \mathrm{mg} / \mathrm{L})$ (Figure 2D). The percentage of MIC $\geq 2 \mathrm{mg} / \mathrm{L}$ is highest in Liaoning (27/38, 71.1\%), and lowest in Shaanxi $(22 / 52,42.3 \%)$ which different to the 8 provinces' overall level $(271 / 451,60.1 \%)$ (Chi-square $=6.06, P<$ 0.05 ). The high-level resistant (MIC $\geq 16 \mathrm{mg} / \mathrm{L}$ ) percentage varied from $3.8 \%-35.5 \%$, with the highest in Guangxi, and lowest in Shaanxi.

\section{Correlation Between MICs of Different Drugs}

Out of all 540 isolates tested, 503 isolates had effective MICs for meropenem, and 540 isolates had effective MICs for other drugs. Analysis of the correlation between doxycycline and ceftriaxone or azithromycin among those isolates with doxycycline $\mathrm{MIC}<16 \mathrm{mg} / \mathrm{L}$. MICs distribution and correlation analyses for meropenem, fosfomycin, berberine hydrochloride, doxycycline with ceftriaxone and azithromycin are shown in Figure 3A-D and Figure 4A-D, respectively, accompanied with its $r$ value and $P$ value. Notably, a correlation of $r=0.50(P<0.001)$ was observed between meropenem and ceftriaxone (Figure 3A). Furthermore, a correlation of $r=0.34(P<0.001)$ was observed between berberine hydrochloride and azithromycin (Figure 4C).

\section{MIC Distribution of Meropenem, Fosfomycin, Berberine hydrochloride, and Doxycycline in DSC or Azithromycin Resistant Isolates}

Out of all 540 tested isolates, 24 isolates were DSC and 87 isolates were resistant to azithromycin. Three isolates were resistant to both ceftriaxone and azithromycin. The MICs distribution of the evaluated drugs for $N$. gonorrhoeae isolates with DSC is demonstrated in Table 2. Among the 24 DSC isolates (Table 2), only 9 isolates showed an MIC of $0.125 \mathrm{mg} / \mathrm{L}$ in meropenem. Table 3 shows MICs' distributions of evaluated drugs for $N$. gonorrhoeae isolates resistant to azithromycin.

\section{Discussion}

The options for effective treatment of N. gonorrhoeae are severely limited by both an increasing resistance of $N$. gonorrhoeae and a limited number of novel antimicrobial agents, and this deficiency poses a significant global healthcare burden. As a result, WHO placed N. gonorrhoeae on a global priority list of antibiotic-resistant pathogens for which to develop novel antimicrobial medicines, ${ }^{27}$ suggesting that finding alternative therapeutics for treating N. gonorrhoeae infections is paramount.

Ceftriaxone and azithromycin dual therapy is recommended for gonorrhea treatment in many countries. After the first high ceftriaxone-resistant $N$. gonorrhoeae isolate H041 was reported in Japan, ${ }^{28}$ an increasing number of ceftriaxone-resistant isolates were reported in other countries such as France, ${ }^{29}$ Australia, ${ }^{30}$ Canada, ${ }^{31}$ and China. ${ }^{32}$ In China, high levels of resistance to azithromycin (18.6\%) and decreased susceptibility to ceftriaxone $(10.8 \%)$ were reported from 2013 to $2016,{ }^{10}$ which indicates the cruciality of searching for alternative treatment drugs.

With broad-spectrum antimicrobial activities, meropenem has been suggested as an alternative therapeutic agent for cephalosporin-resistant $N$. gonorrhoeae in vitro, ${ }^{16}$ and ertapenem, another carbapenem antibiotic similar to meropenem but with a relatively narrow antimicrobial spectrum, cured patients who failed treatment with ceftriaxone $1 \mathrm{~g}$ in the $\mathrm{UK}^{33}$ In this study, meropenem showed lowlevel $\mathrm{MIC}_{50}$ and $\mathrm{MIC}_{90}$ values. $\mathrm{MIC}_{50}$ of meropenem was similar to that of ceftriaxone, while $\mathrm{MIC}_{90}$ was $1 / 2$-fold lower (data not shown). Using the MIC interpretation of meropenem according to CLSI breakpoint (CLSI M100S30) in testing and reporting for Escherichia coli to interpret MIC data for meropenem (MIC $\leq 1 \mathrm{mg} / \mathrm{L}$ ), all isolates 


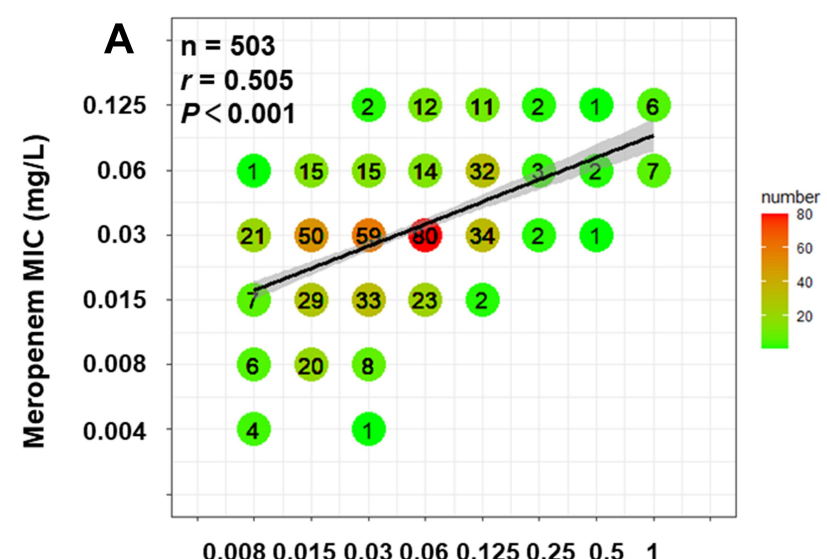

Ceftriaxone MIC (mg/L)

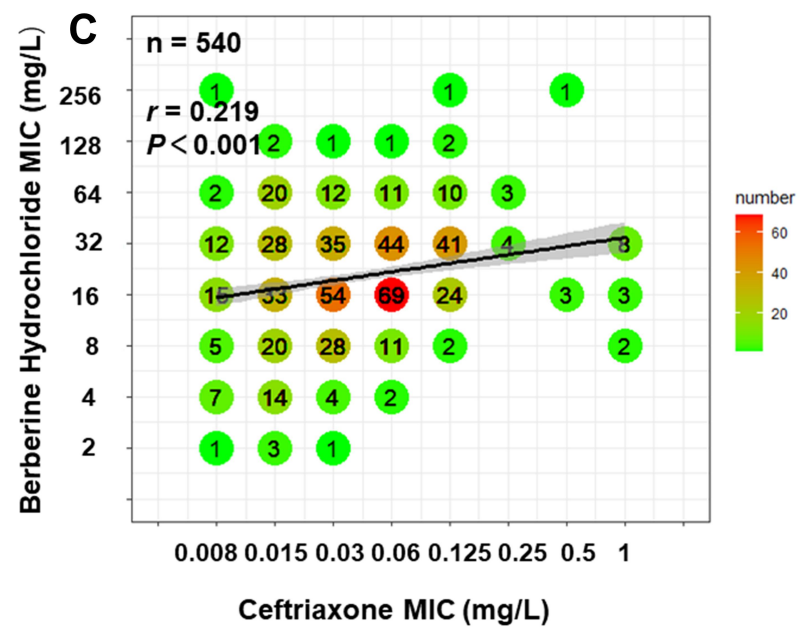

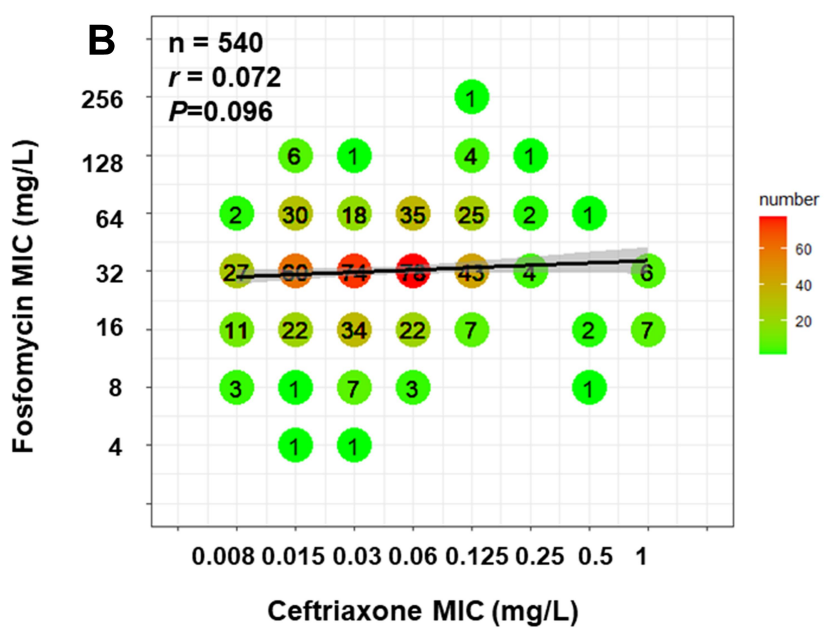

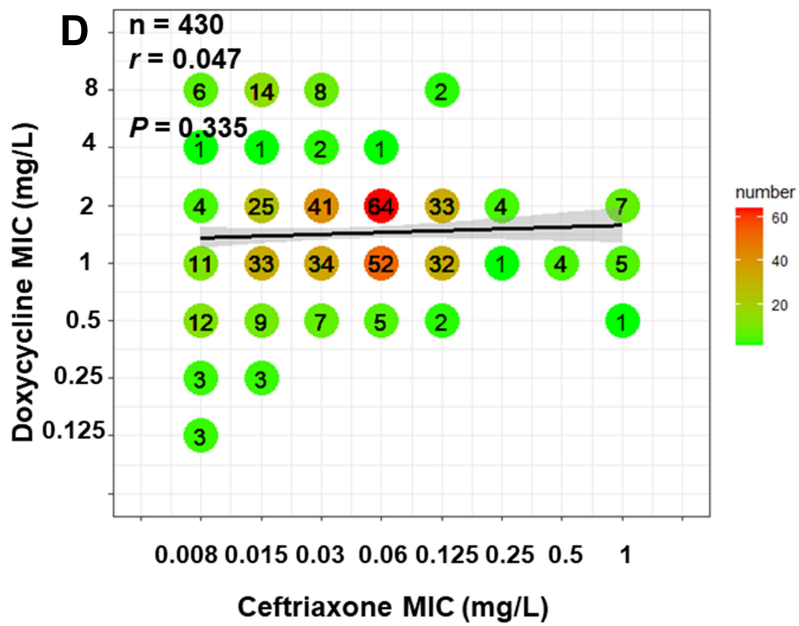

Figure 3 (A-D) Correlation between MICs of meropenem, fosfomycin, berberine hydrochloride, doxycycline and ceftriaxone. Each symbol represents one or multiple isolates, which is indicated by specific numbers and colors. The regression line was calculated using $\log _{2}$-transformed MIC data. $r$ value and its corresponding $P$ value are indicated. For MICs determined as $\leq$ or $\geq$ a specific value, this specific value was used for calculating linear regression of log ${ }_{2}$-transformed MIC data.

were susceptible to meropenem. In spite of a positive correlation between meropenem $\mathrm{MIC}$ and ceftriaxone MIC (Figure 3A), the MIC value of meropenem remained lower than that of ceftriaxone among the DSC isolates (Table 2). All 24 DSC isolates showed meropenem MIC $<0.25 \mathrm{mg} / \mathrm{L}$. Although clinicians are cautious about using carbapenem antibiotics, the increasing number of patients facing either ceftriaxone-resistant or simply persistent gonorrhea may warrant meropenem's clinical use. Our in vitro data suggests that meropenem might offer potential over DSC gonococcal infections treatment.

Fosfomycin has great advantages in urethritis treatment because of its high concentrations in urine. Peak fosfomycin concentrations in urine reached $4415 \mathrm{mg} / \mathrm{L}$ within the first 4 hrs, while concentrations remained above the MIC for up to 3 days following $3 \mathrm{~g}$ oral administration. ${ }^{34}$ Moreover, it is taken orally and has a high oral bioavailability as well as excellent safety. $^{35}$ Fosfomycin exhibits bactericidal activity through a unique mechanism of action to which a variety of organisms, including $N$. gonorrhoeae, are susceptible. Here, the $\mathrm{MIC}_{50}$ and $\mathrm{MIC}_{90}$ of fosfomycin were $32 \mathrm{mg} / \mathrm{L}$ and $64 \mathrm{mg} / \mathrm{L}$, respectively. The fosfomycin MIC of $N$. gonorrhoeae isolates from the period 2011-2012 and 2015-2017 in China Zhejiang province was evaluated, and the $\mathrm{MIC}_{90}$ was $32 \mathrm{mg} / \mathrm{L}{ }^{36}$ The $\mathrm{MIC}_{90}$ reported in this study may be higher than that $(16 \mathrm{mg}$ / L) reported in a research study where 89 isolates were collected from 1998 to 2014, and different antimicrobial susceptibility methods were used. ${ }^{35}$ In the 1970s, it was found that patients with gonococcal urethritis demonstrated a $90 \%$ clinical and bacteriological success rate associated with a fosfomycin MIC of $\leq 64 \mathrm{mg} / \mathrm{L} .{ }^{37}$ Since no susceptibility criteria of fosfomycin for $N$. gonorrhoeae has been established, interpretation 

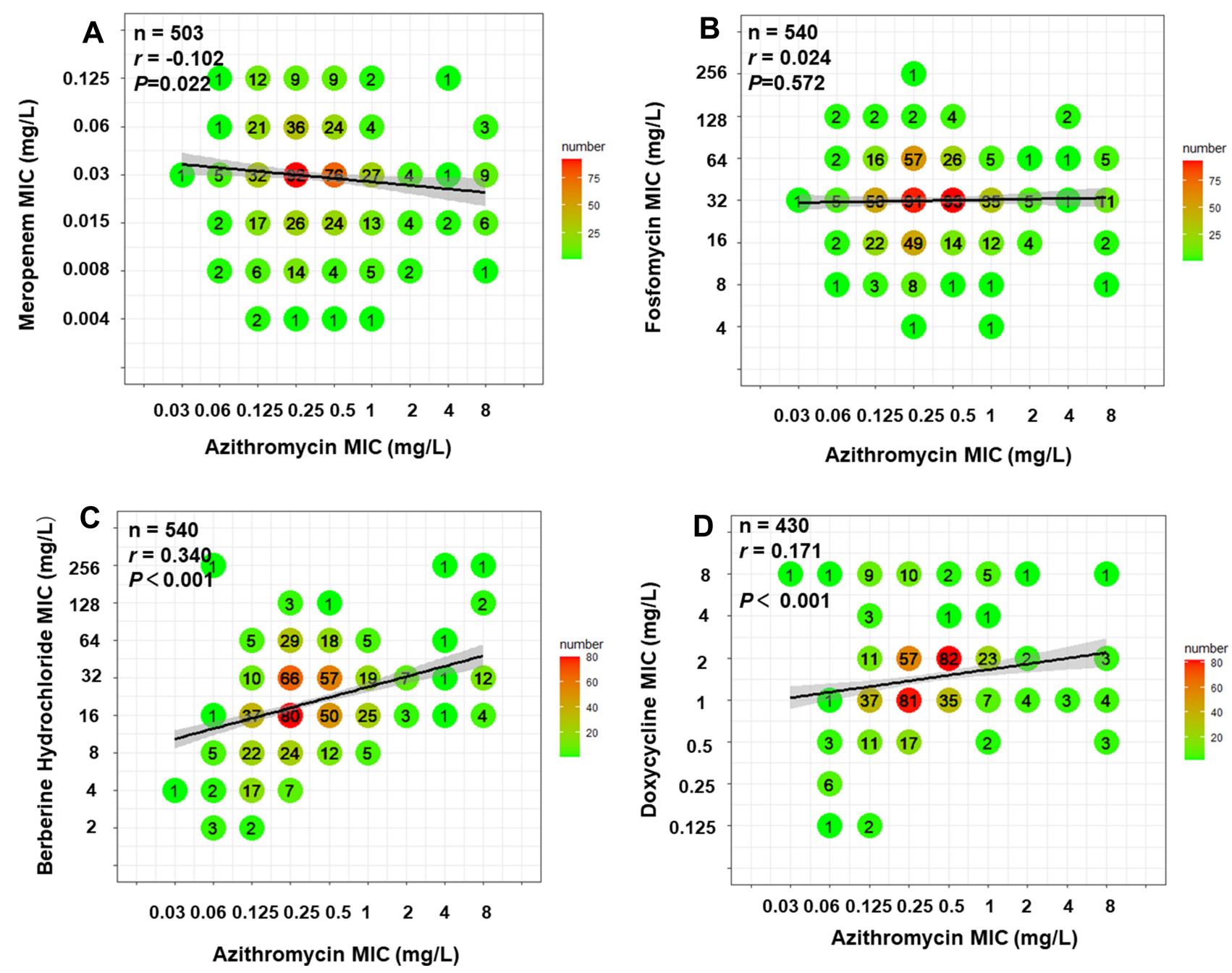

Figure 4 (A-D) Correlation between MICs of meropenem, fosfomycin, berberine hydrochloride, doxycycline and azithromycin. Each symbol represents one or multiple isolates, which is indicated by specific numbers and colors. The regression line was calculated using $\log _{2}$-transformed MIC data. $r$ value and its corresponding $P$ value are indicated. For MICs determined as $\leq$ or $\geq$ a specific value, this specific value was used for calculating linear regression of log ${ }_{2}$-transformed $\mathrm{MIC}$ data.

according to CLSI breakpoint only approved in testing and reporting for Escherichia coli and Enterococcus faecalis urinary isolates (MIC $\leq 64 \mathrm{mg} / \mathrm{L}$, susceptible; $128 \mathrm{mg} / \mathrm{L}$, intermediately susceptible; MIC $\geq 256 \mathrm{mg} / \mathrm{L}$, resistant), it was found that only 1 isolate $(0.2 \%)$ showed resistance while 13 isolates (2.9\%) showed intermediate susceptibility in the 8 provinces. No significant correlations between fosfomycin MICs and ceftriaxone MICs or azithromycin MICs were found (Figure 4C), implying the limited overlap in resistant mechanisms between fosfomycin and ceftriaxone or azithromycin and indicating its potential as an alternative. Further, 23/24 DSC isolates showed susceptible (MIC $\leq 64 \mathrm{mg} / \mathrm{L}$ ) to fosfomycin suggesting it could be used as an alternative antimicrobial for the treatment of DSC infections. In this study, we used CLSI breakpoint (CLSI M100-S30, MIC $\leq 64 \mathrm{mg} / \mathrm{L}$, susceptible; $128 \mathrm{mg} / \mathrm{L}$, intermediately susceptible; MIC $\geq 256 \mathrm{mg} / \mathrm{L}$, resistant) instead of EUCAST breakpoint (MIC $\leq 32 \mathrm{mg} / \mathrm{L}$, susceptible; MIC > 32mg/L, resistant) set for Enterobacteriaceae $^{38}$ to interpret our susceptibility data. However, the percentage of resistant isolates may be greater if EUCAST breakpoint was referenced, demonstrating a discrepancy due to the use of different criterion. Establishing susceptibility breakpoints for $N$. gonorrhoeae is important in advancing our understanding of appropriate doses of fosfomycin to treat gonorrhea. Based on our data as well as pharmacokinetics and pharmacodynamics (PK/PD) data reported previously, fosfomycin may be a good candidate for further study. However, further studies are needed to evaluate its efficacy.

Berberine, an isoquinoline alkaloid extracted from Coptis chinensis, has been reported to have antimicrobial activity against bacteria, fungi, viruses, and Chlamydia. ${ }^{24,39}$ Anti 

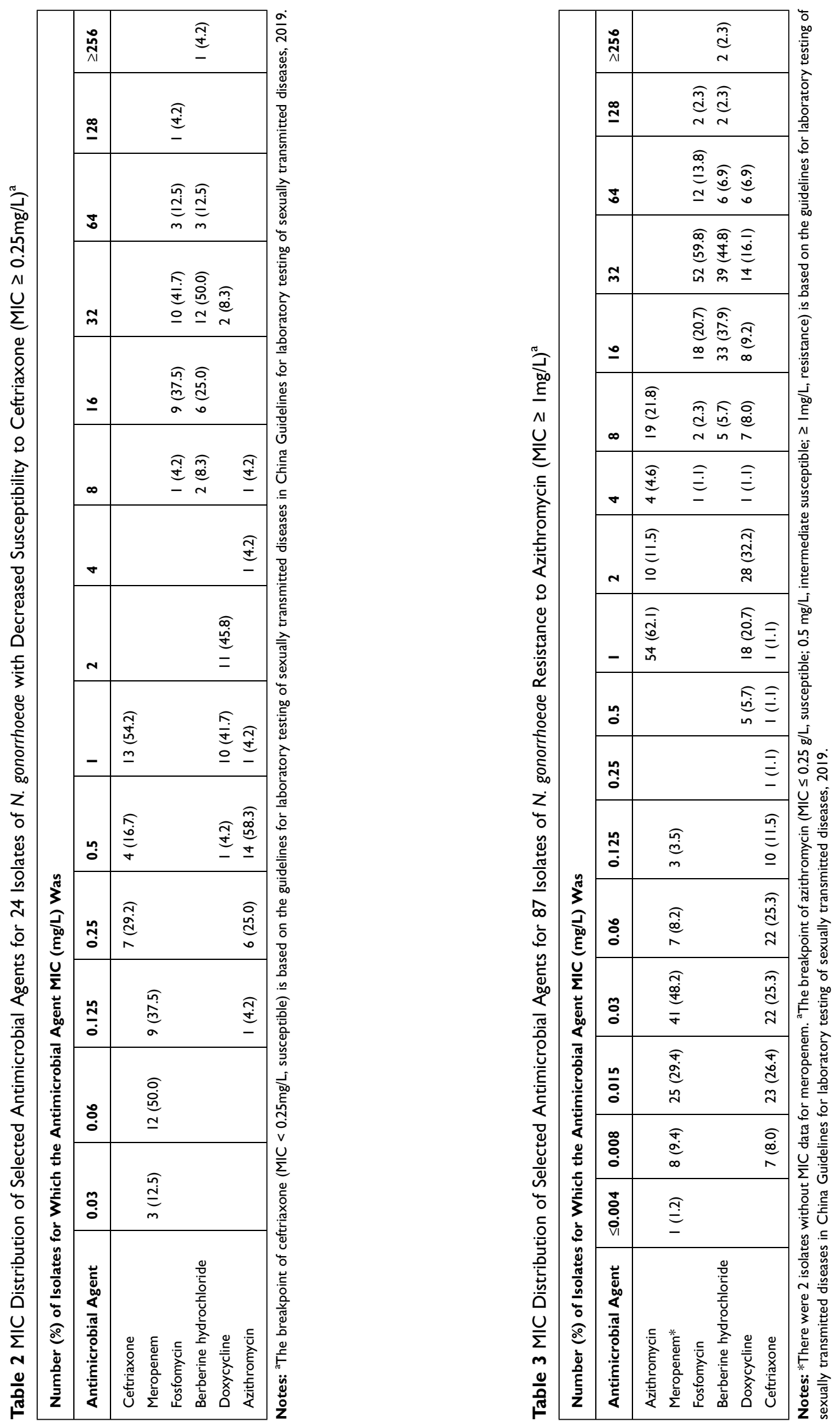
$N$. gonorrhoeae activity of berberine has also been reported. $^{40,41}$ A previous study with a panel of 28 $N$. gonorrhoeae isolates showed that berberine inhibited the growth of penicillinase-producing, spectinomycin-resistant and susceptible $N$. gonorrhoeae isolates. ${ }^{40}$ Berberine hydrochloride is a commonly used clinical drug which is mainly administered orally and used externally. Berberine has poor intestinal absorption which is related to P-glycoprotein. ${ }^{42}$ The PK/PD of berberine in rat model shows the concentration in plasma is low, while the digestive tract and tissue concentrations were higher than that in plasma. ${ }^{43}$ In this study, among 451 isolates, 8 isolates (1.8\%) exhibited the $\mathrm{MIC}>64 \mathrm{mg} / \mathrm{L}$. The $\mathrm{MIC}_{90}$ in this study is lower than MIC reported before in other bacteria such as Escherichia coli, Staphylococcus aureus, Pseudomonas aeruginosa ${ }^{44}$ and so on. In our study, berberine hydrochloride showed potential anti-gonococcal activity and a correlation with azithromycin $(r=0.34, P<0.001)$ and ceftriaxone $(r=0.21, P<0.001)$. And in 24 DSC isolates and 87 azithromycin resistant isolates, the percentage of isolates with berberine hydrochloride $\mathrm{MIC} \leq 64 \mathrm{mg} / \mathrm{L}$ were $95.8 \%$ and $95.4 \%$, respectively. Based on this study, berberine hydrochloride shows antigonococcal effects in vitro. However, its potential use as a potential therapeutic agent should be further studied.

Although tetracyclines were removed from monotherapy for gonorrhea treatment due to the rapid spread of resistant strains, doxycycline was recommended to treat gonorrhea that does not exclude chlamydia infection or gonococcal pelvic inflammatory disease in the Chinese Guidelines for diagnosis and treatment of syphilis, gonorrhea, and genital Chlamydia trachomatis infection (2020). ${ }^{3}$ The USA Sexually Transmitted Diseases Treatment Guidelines, 2015, also proposed doxycycline as a replacement for azithromycin in patients with an azithromycin allergy. ${ }^{4}$ A recent survey investigating clinicians' drug choices when treating gonorrhea in China showed that 148 of 1890 clinicians (7.8\%) used doxycycline in gonorrhea treatment (unpublished data). However, in China, the data of doxycycline susceptibility on $N$. gonorrhoeae is limited. A study on gonococcal isolates in China Zhejiang province showed that the $\mathrm{MIC}_{90}$ of doxycycline is $16 \mathrm{mg} / \mathrm{L}$, and in our study, it was the same as the 8 provinces overall level but different to Liaoning ( $32 \mathrm{mg} / \mathrm{L}$ ), Guangxi (32 $\mathrm{mg} / \mathrm{L})$, Sichuan(32 $\mathrm{mg} / \mathrm{L})$ and Shaanxi $(2 \mathrm{mg} / \mathrm{L})$. Moreover, our study showed 271 isolates $(271 / 451,60.1 \%)$ with doxycycline MIC $>1 \mathrm{mg} / \mathrm{L}$ (which is the breakpoint in tetracycline for reference), and this varied from $42.3 \%$ to $71.1 \%$ in different provinces. Based on the high-level MIC of doxycycline identified in this study, doxycycline is not a highly recommended treatment for gonorrhea in China. Interestingly, our study showed few high-level doxycycline resistant strains in Shaanxi, which indicated the geographical variations on MIC distributions. This emphasizes the importance of antimicrobial susceptibility surveillance in different provinces in order to select high sensitivity therapeutic drugs in clinical treatment according to local antimicrobial susceptibility surveillance data. However, previous studies show that among patients with gonococcal infections, $46 \%$ were also infected with genital Chlamydia trachomatis, ${ }^{45}$ the primary treatment of which remains doxycycline. Our in vitro data suggests that doxycycline may also be effective against some sensitive $N$. gonorrhoeae infection in patients co-infected with Chlamydia trachomatis when treating Chlamydia trachomatis infection.

Due to China's large population and territory, the living geographical position of citizens in different areas may have different medication and lifestyle characteristics. We analyzed the variation of antimicrobial susceptibility among domestic provinces and confirmed distinguishing MIC distribution (Figure 2). This indicated the geographical variations and raised more concerns about potential resistance differences in specific locations. Antimicrobial resistance surveillance should be strengthened in various provinces to provide a basis for the establishment of guidelines in gonorrhea treatment. At the same time, this research may provide a basis for monitoring antimicrobial susceptibility in the The Belt and Road Initiative countries such as Mongolia.

The current study is the first nationwide research evaluating the $N$. gonorrhoeae susceptibility to meropenem, fosfomycin, berberine hydrochloride and doxycycline in China. This study was a national surveillance of meropenem, fosfomycin, berberine hydrochloride and doxycycline susceptibility with isolates collected from eight provinces in China, but the isolates are only part of China-GRSP, and the isolates account for only $0.3 \%$ (451/ 133,156) of reported cases of gonorrhea in 2018 suggesting limitations to the scope of this study. Moreover, less than a quarter of the 34 provinces and areas in the country participated in the study, and the provinces distribution (Figure 1) in our study may also lead to a potential bias. Also, all the isolates were from the genitourinary tract, and the lack of rectal and pharyngeal specimens may also lead to a bias on antimicrobial sensitivity. Though breakpoints in other bacteria or similar drugs were considered in the interpretation of these data, they may not translate well for gonorrhea due to different pharmacokinetic parameters involved in genital and extragenital infections. In addition, this is only an in vitro experiment to explore the effectiveness of these drugs, the specific efficacy may need further investigation. 


\section{Conclusion}

The study evaluated meropenem, fosfomycin, berberine hydrochloride, and doxycycline MICs of $N$. gonorrhoeae isolates in 2018 from 8 provinces in China and analyzed the correlation between drugs to predict the potential cross-resistance. The performance of these drugs on DSC and azithromycin resistant isolates was also evaluated. Our results suggest that meropenem might be a promising treatment option for resistant gonococcal infections, while the effect of fosfomycin and berberine hydrochloride needed further research. However, doxycycline is not suitable for monotherapy in treating gonorrhea in China. Though breakpoints in other bacteria or similar drugs were considered in the interpretation of these data, they may not translate well for $N$. gonorrhoeae infections. Overall, this study provides the antimicrobial sensitivity of these drugs to $N$. gonorrhoeae isolates newly collected in eight provinces from China, adding new information on potential alternative medicines to treat gonorrhea. To continue evaluating their effectiveness, future studies are needed.

\section{Acknowledgments}

The authors thank these patients for providing the specimens and acknowledge LF Yuan, WL Cao, BY Zhu, ZG Zhang, G Yong, CS Zhu, ZJ Zheng and L Bai and their participants for isolates collecting. We thank $\mathrm{H} \mathrm{Hu}$ for his technology support in making part of the figures.

\section{Funding}

This work was supported by the National Science and Technology Major Project (2018ZX101010 01-004-003), the Chinese Academy Medical Sciences Initiative Medicine (2016-I2M-3-021), Fundamental Research Funds for the Central Universities (3332018122), and Jiangsu Province Youth Fund Project (BK20180156). The sponsors had no role in study design, data collection and analysis, data interpretation, mainuscript preparation, and the decision for publication.

\section{Disclosure}

The authors report no conflicts of interest in this work.

\section{References}

1. Rowley J, Vander Hoorn S, Korenromp E, et al. Chlamydia, gonorrhoea, trichomoniasis and syphilis: global prevalence and incidence estimates, 2016. Bull World Health Organ. 2019;97(8):548-562P. doi:10.2471/BLT.18.228486

2. National Health Commission of the PRC [homepage on Internet]. Overview of the national epidemic of infectious diseases in 2019 2020. Available from: http://www.nhc.gov.cn/jkj/s3578/202004/ b1519e1bc1a944fc8ec176db600f68d1.shtml. Accessed June 13, 2020
3. National Center for STD Control, Chinese Center for Disease Control and Prevention; Venereology Group, Chinese Society of Dermatology; Subcommittee on Venereology, China Dermatologist Association. Guidelines for diagnosis and treatment of syphilis, gonorrhea and genital Chlamydia trachomatis infection (2020). Chin $J$ Dermatol. 2020;53(3):168-179.

4. Workowski KA, Bolan GA; Centers for Disease C, Prevention. Sexually transmitted diseases treatment guidelines, 2015. MMWR Recomm Rep. 2015;64(RR-03):1-137.

5. Australian Sexual Health Alliance. Australian STI management guidelines for use in primary care; 2019; Available from: http:// www.sti.guidelines.org.au/sexually-transmissible-infections/gonor rhoea\#management. Accessed September 27, 2020.

6. Public Health Agency of Canada. Canadian guidelines on sexually transmitted infections; 2017. Available from: http://publications.gc. ca/collections/collection_2017/aspc-phac/HP40-1-2017-2-eng.pdf. Accessed September 27, 2020.

7. Unemo M. The 2012 European guideline on the diagnosis and treatment of gonorrhoea in adults recommends dual antimicrobial therapy. Eur Surveill. 2012;17(47).

8. The New Zealand Sexual Health Society. New Zealand guideline for the management of gonorrhoea, 2014, and response to the threat of antimicrobial resistance; 2014. Available from: https://www.nzshs. org/docman/guidelines/best-practice-guidelines/142-new-zealandguideline-for-the-management-of-gonorrhoea-2014-and-response-tothe-threat-of-antimicrobial-resistance/file. Accessed September 27, 2020.

9. World Health Organization [homepage on Internet]. WHO guidelines for the treatment of Neisseria gonorrhoeae; 2016. Available from: http://apps.who.int/iris/bitstream/10665/246114/1/9789241549691eng.pdf?ua=1. Accessed June 13, 2020.

10. Yin YP, Han Y, Dai XQ, et al. Susceptibility of Neisseria gonorrhoeae to azithromycin and ceftriaxone in China: a retrospective study of national surveillance data from 2013 to 2016. PLoS Med. 2018;15(2):e1002499. doi:10.1371/journal.pmed.1002499

11. Yin YP. Guidelines for laboratory testing of sexually transmitted diseases. Beijing: People's Medical Publishing House (PMPH); 2019.

12. Liu JW, Xu WQ, Zhu XY, et al. Gentamicin susceptibility of Neisseria gonorrhoeae isolates from 7 provinces in China. Infect Drug Resist. 2019;12:2471-2476. doi:10.2147/IDR.S214059

13. World Health Organization [homepage on Internet]. Global action plan to control the spread and impact of antimicrobial resistance in Neisseria gonorrhoeae; 2012. Available from: https://www.who.int/ reproductivehealth/publications/rtis/9789241503501/en/. Accessed May 10, 2020.

14. Xiao Y, Li L. China's national plan to combat antimicrobial resistance. Lancet Infect Dis. 2016;16(11):1216-1218. doi:10.1016/ S1473-3099(16)30388-7

15. Chen XS, Yin YP, Li XY. A ROADMAP plan to address research needs for gonococcal antimicrobial resistance in China. Clin Infect Dis. 2019;68(3):505-510. doi:10.1093/cid/ciy566

16. Hiyama Y, Takahashi S, Sato T, et al. Evaluation of susceptibilities to carbapenems and faropenem against cephalosporin-resistant neisseria gonorrhoeae clinical isolates with penA mosaic alleles. Microbial Drug Resist (Larchmont, NY). 2019;25(3):427-433. doi:10.1089/ mdr.2018.0263

17. Tesh LD, Shaeer KM, Cho JC, et al. Neisseria gonorrhoeae and fosfomycin: past, present and future. Int $J$ Antimicrob Agents. 2015;46(3):290-296. doi:10.1016/j.ijantimicag.2015.05.007

18. Yuan Z, He C, Yan S, Ke Y, Tang W. Randomized controlled clinical trial on the efficacy of fosfomycin trometamol for uncomplicated gonococcal urethritis in men. Clin Microbiol. 2016;22(6):507-512. doi:10.1016/j.cmi.2016.03.031

19. Yuan Y, Si S, Jiang J. Antibacterial activity of berberine. Acta Pharmaceutica Sinica. 2018;53:163-168. 
20. Wu LT, Tsou MF, Ho CC, Chuang JY, Kuo HM, Chung JG. Berberine inhibits arylamine $\mathrm{N}$-acetyltransferase activity and gene expression in Salmonella typhi. Curr Microbiol. 2005;51(4):255-261. doi:10.1007/ s00284-005-4569-7

21. Domadia PN, Bhunia A, Sivaraman J, Swarup S, Dasgupta D. Berberine targets assembly of Escherichia coli cell division protein FtsZ. Biochemistry. 2008;47(10):3225-3234. doi:10.1021/bi7018546

22. Boberek JM, Stach J, Good L. Genetic evidence for inhibition of bacterial division protein FtsZ by berberine. PLoS One. 2010;5(10):e13745.

23. Sun D, Abraham SN, Beachey EH. Influence of berberine sulfate on synthesis and expression of Pap fimbrial adhesin in uropathogenic Escherichia coli. Antimicrob Agents Chemother. 1988;32 (8):1274-1277. doi:10.1128/AAC.32.8.1274

24. Anonymous. Berberine. Alter Med Rev. 2000;5(2):175-177.

25. Imanshahidi M, Hosseinzadeh $H$. Pharmacological and therapeutic effects of Berberis vulgaris and its active constituent, berberine. Phytother Res. 2008;22(8):999-1012.

26. Unemo M, Golparian D, Sánchez-Busó L, et al. The novel 2016 WHO Neisseria gonorrhoeae reference strains for global quality assurance of laboratory investigations: phenotypic, genetic and reference genome characterization. J Antimicrob Chemother. 2016;71 (11):3096-3108. doi:10.1093/jac/dkw288

27. Tacconelli E, Carrara E, Savoldi A, et al. Discovery, research, and development of new antibiotics: the WHO priority list of antibiotic-resistant bacteria and tuberculosis. Lancet Infect Dis. 2018;18(3):318-327. doi:10.1016/S1473-3099(17)30753-3

28. Ohnishi M, Golparian D, Shimuta K, et al. Is Neisseria gonorrhoeae initiating a future era of untreatable gonorrhea?: detailed characterization of the first strain with high-level resistance to ceftriaxone. Antimicrob Agents Chemother. 2011;55(7):3538-3545. doi:10.1128/ AAC.00325-11

29. Unemo M, Golparian D, Nicholas R, Ohnishi M, Gallay A, Sednaoui P. High-level cefixime- and ceftriaxone-resistant Neisseria gonorrhoeae in France: novel penA mosaic allele in a successful international clone causes treatment failure. Antimicrob Agents Chemother. 2012;56(3):1273-1280. doi:10.1128/AAC.05760-11

30. Lahra MM, Ryder N, Whiley DM. A new multidrug-resistant strain of Neisseria gonorrhoeae in Australia. $N$ Engl J Med. 2014;371 (19):1850-1851. doi:10.1056/NEJMc1408109

31. Lefebvre B, Martin I, Demczuk W, et al. Ceftriaxone-resistant Neisseria gonorrhoeae, Canada, 2017. Emerg Infect Dis. 2018;24 (2):381-383. doi:10.3201/eid2402.171756

32. Chen SC, Yin YP, Chen XS. Cephalosporin-resistant Neisseria gonorrhoeae clone, China. Emerg Infect Dis. 2018;24(4):804-806. doi:10.3201/eid2404.171817

33. Eyre DW, Town K, Street T, et al. Detection in the United Kingdom of the Neisseria gonorrhoeae FC428 clone, with ceftriaxone resistance and intermediate resistance to azithromycin, October to December 2018. Eur Surveill. 2019;24(10).
34. Reffert JL, Smith WJ. Fosfomycin for the treatment of resistant gram-negative bacterial infections. Insights from the Society of Infectious Diseases Pharmacists. Pharmacotherapy. 2014;34 (8):845-857. doi:10.1002/phar.1434

35. Hauser C, Hirzberger L, Unemo M, Furrer H, Endimiani A. In vitro activity of fosfomycin alone and in combination with ceftriaxone or azithromycin against clinical Neisseria gonorrhoeae isolates. Antimicrob Agents Chemother. 2015;59(3):1605-1611. doi:10.1128/ AAC.04536-14

36. Yang F, Yan J, Zhang J, van der Veen S. Evaluation of alternative antibiotics for susceptibility of gonococcal isolates from China. Int $J$ Antimicrob Agents. 2020;55(2):105846. doi:10.1016/j. ijantimicag.2019.11.003

37. Rodríguez A, Gallego A, Olay T, Mata JM. Bacteriological evaluation of fosfomycin in clinical studies. Chemotherapy. 1977;23(Supp1 1):247-258. doi:10.1159/000222055

38. The European Committee on Antimicrobial Susceptibility Testing. European committee on antimicrobial susceptibility testing breakpoint tables for interpretation of MICs and zone diameters version 10.0; 2020; Available from: https://www.eucast.org/fileadmin/src/ media/PDFs/EUCAST_files/Breakpoint_tables/v_10.0_Breakpoint_ Tables.pdf. Accessed May 10, 2020.

39. Vuddanda PR, Chakraborty S, Singh S. Berberine: a potential phytochemical with multispectrum therapeutic activities. Expert Opin Investig Drugs. 2010;19(10):1297-1307. doi:10.1517/13543784.2010.517745

40. Cybulska P, Thakur SD, Foster BC, et al. Extracts of Canadian first nations medicinal plants, used as natural products, inhibit neisseria gonorrhoeae isolates with different antibiotic resistance profiles. Sex Transm Dis. 2011;38(7):667-671. doi:10.1097/OLQ.0b013e31820cb166

41. Li G, Chen Q, Chen J. In vitro bacteriostatic effects of 21 Chinese herbs on gonorrhea strains. J Clin Dermatol. 2003;334.

42. Pan G-Y, Wang G-J, Liu X-D, Fawcett JP, Xie -Y-Y. The involvement of P-glycoprotein in berberine absorption. Pharmacol Toxicol. 2002;91(4):193-197. doi:10.1034/j.1600-0773.2002.t01-1-910403.x

43. Tan XS, Ma JY, Feng R, et al. Tissue distribution of berberine and its metabolites after oral administration in rats. PLoS One. 2013;8(10): e77969. doi:10.1371/journal.pone.0077969

44. Cernáková M, Kostálová D. Antimicrobial activity of berberine-a constituent of Mahonia aquifolium. Folia Microbiol (Praha). 2002;47 (4):375-378. doi:10.1007/BF02818693

45. Datta SD, Sternberg M, Johnson RE, et al. Gonorrhea and chlamydia in the United States among persons 14 to 39 years of age, 1999 to 2002. Ann Intern Med. 2007;147(2):89-96. doi:10.7326/0003-4819147-2-200707170-00007
Infection and Drug Resistance

\section{Publish your work in this journal}

Infection and Drug Resistance is an international, peer-reviewed openaccess journal that focuses on the optimal treatment of infection (bacterial, fungal and viral) and the development and institution of preventive strategies to minimize the development and spread of resistance. The journal is specifically concerned with the epidemiology of antibiotic resistance and the mechanisms of resistance development and diffusion in both hospitals and the community. The manuscript management system is completely online and includes a very quick and fair peerreview system, which is all easy to use. Visit http://www.dovepress.com/ testimonials.php to read real quotes from published authors. 